\title{
Nutrition in Pregnant Immigrant Nigerian Women
}

\author{
K. L. Lindsay ${ }^{1}$, E. R. Gibney ${ }^{2}$, B. McNulty ${ }^{2}$ and F. A. McAuliffe ${ }^{1}$ \\ ${ }^{1}$ UCD Obstetrics and Gynaecology, National Maternity Hospital, Holles Street, Dublin 2, ${ }^{2}$ UCD School of Agriculture, \\ Food Science and Veterinary Medicine, UCD, Dublin, Republic of Ireland
}

Immigrant women from Sub-Saharan Africa (SSA) are reported to have poor pregnancy outcomes, with high rates of preterm delivery, low birth weight and perinatal mortality ${ }^{(1)}$. However, the relationship between such outcomes and the nutritional status of these women is unknown. Nigerians are the most common SSA immigrant group in many Western countries, including the UK and Ireland. There is a paucity of literature investigating nutritional intakes among Nigerian immigrant women, whether pregnant or non-pregnant. The aim of the current study is to establish the nutritional status and dietary intakes of pregnant immigrant Nigerian women.

Fifty-two Nigerian women in their $2^{\text {nd }}$ or $3^{\text {rd }}$ trimester of pregnancy were recruited from ante-natal clinics at the National Maternity Hospital, Dublin. Written informed consent was obtained. Anthropometric details were obtained from patient charts and nutritional supplement usage was recorded. Dietary intakes were assessed using a single 24-hour recall and data was entered into WISP for nutrient intake analysis. Population and individual mean nutrient intakes were compared to the Food Safety Authority of Ireland (FSAI) values for Recommended Dietary Allowances (RDA) ${ }^{(2)}$.

Energy and macronutrient intakes were adequate and compliant to dietary guidelines when expressed as a percentage of total energy (TE). Individual compliance to micronutrient RDAs from diet plus supplements was inadequate for calcium, vitamin D, vitamin A and folate. When population mean intakes $(n=52)$ are compared to the RDAs, calcium intake remains low and a small portion of women still fail to achieve the recommended vitamin D intake. Reported usage of supplements containing vitamin D and folic acid during pregnancy was $40 \%$ and $79 \%$, respectively. Only eight women (15\%) took folic acid pre-conception.

\begin{tabular}{|c|c|c|c|c|c|c|}
\hline Energy \& Macronutrients & Mean & SD & Micronutrients & $\begin{array}{c}\text { Mean intake } \\
\text { (diet \& supplements) }\end{array}$ & $\begin{array}{l}\text { \% Individuals } \\
\text { meeting RDA }\end{array}$ & $\begin{array}{c}\% \text { Population with } \\
\text { mean intake meeting RDA }\end{array}$ \\
\hline Energy (Kcal) & 2211 & 846 & Calcium (mg) & 694.5 & 7.7 & 25.0 \\
\hline Protein (\%TE) & 18.9 & 8.4 & Iron (mg) & 69.6 & 75.0 & 100 \\
\hline CHO (\% TE) & 52.7 & 9.2 & Vitamin A $(\mu \mathrm{g})$ & 1402.3 & 67.3 & 100 \\
\hline Total fat (\% TE) & 32.9 & 8.0 & Vitamin D $(\mu \mathrm{g})$ & 8.5 & 48.1 & 82.7 \\
\hline SFA (\%TE) & 10.3 & 3.8 & Vitamin $B_{12}(\mu \mathrm{g})$ & 8.8 & 94.2 & 100 \\
\hline MUFA (\%TE) & 11.3 & 3.7 & Vitamin C (mg) & 231.7 & 88.5 & 100 \\
\hline PUFA (\%TE) & 6.8 & 3.6 & Folate $(\mu \mathrm{g})$ & 575.8 & 67.3 & 100 \\
\hline
\end{tabular}

CHO, carbohydrate; SFA, saturated fat; MUFA, monounsaturated fat; PUFA, polyunsaturated fat.

The reported energy and macronutrient intakes are compliant to healthy eating guidelines and only saturated fat intake is marginally higher than recommended. Calcium intakes were particularly inadequate when compared to the RDA, on both the individual and population level. This may be due to a combination of low dietary intakes and the absence of calcium in the commonly used pregnancy multivitamin/mineral supplements. Among $17.3 \%$ of the population, vitamin D intakes were below the RDA, even when supplement use is considered. This group is likely to be at high risk of vitamin D deficiency given their dark skin pigmentation and limited sunlight exposure in Ireland. Although folate intake appears adequate at the time of assessment, pre-conception use of folic acid was poor, increasing the risk of sub-optimal folate status in the critical stage of early pregnancy. Further research into the dietary intakes of pregnant SSA women is required and the relationship between nutritional status and adverse pregnancy outcomes in this group needs to be determined.

This work was supported by the Health Research Board, Ireland.

1. Lindsay KL, Gibney ER \& McAuliffe FA (2012) J Hum Nutr Diet. (In press).

2. Food Safety Authority of Ireland (1999) Recommended Dietary Allowances for Ireland. FSAI: Dublin. 